\title{
ORIGINAL ARTICLE Long-bone fractures in persons with spinal cord injury
}

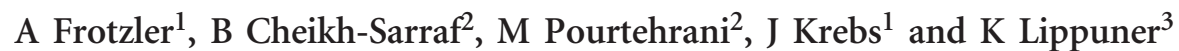

Study design: Retrospective data analysis.

Objectives: To document fracture characteristics, management and related complications in individuals with traumatic spinal cord injury $(\mathrm{SCl})$.

Setting: Rehabilitation centre for SCl individuals.

Method: Patients' records were reviewed. Patients with traumatic $\mathrm{SCl}$ and extremity fractures that had occurred after $\mathrm{SCl}$ were included. Patient characteristics, fractured bone, fracture localisation, severity and management (operative/conservative), and fracturerelated complications were extracted.

Results: A total of 156 long-bone fractures in $107 \mathrm{SCl}$ patients (34 women and 73 men) were identified. The majority of patients were paraplegics (77.6\%) and classified as American Spinal Injury Association Impairment Scale A (86.0\%). Only the lower extremities were affected, whereby the femur (60.9\% of all fractures) was fractured more frequently than the lower leg (39.1\%). A total of 70 patients $(65.4 \%)$ had one fracture, whereas 37 patients $(34.6 \%)$ had two or more fractures. Simple or extraarticular fractures were most common (75.0\%). Overall, 130 (83.3\%) fractures were managed operatively. Approximately half of the femur fractures (48.2\%) were treated with locking compression plates. In the lower leg, fractures were mainly managed with external fixation (48.8\%). Conservative fracture management was applied in $16.7 \%$ of the cases and consisted of braces or a well-padded soft cast. Fracture-associated complications were present in $13.5 \%$ of the cases but did not differ significantly between operative (13.1\%) and conservative (15.4\%) fracture management.

Conclusion: SCl was associated with simple or extraarticular fractures of the distal femur and the lower leg. Fractures were mainly managed operatively with a low complication rate.

Spinal Cord (2015) 53, 701-704; doi:10.1038/sc.2015.74; published online 19 May 2015

\section{INTRODUCTION}

Long-bone fractures are a common secondary condition of spinal cord injury (SCI). An average fracture incidence of $2.2 \%$ per year with a mean time to first fracture after traumatic SCI of 8.9 years has been reported. ${ }^{1}$ The lower extremities are predominantly affected by fractures, and fractures are typically located in the diaphyseal or distal femur and in the proximal lower leg. ${ }^{2-4}$ Typically, SCI individuals suffer from fragility fractures, that is, low-energy fractures. ${ }^{5}$ The extensive loss of bone mass in the paralysed extremities is a main factor contributing to the increased fracture risk after SCI. ${ }^{1,5,6}$ Within the first years after motor complete SCI, bone mineral density decreases by $50-70 \%$ in the lower extremities. ${ }^{7,8}$ This loss of bone density is paralleled by an overall increased fracture rate after SCI, which is twice the fracture rate in the able-bodied population. ${ }^{5}$ Spinal cord-injured women have been reported to have a 1.6 times higher risk of long-bone fractures compared with spinal cord-injured men. This seems to be the result of the higher degree of bone loss in SCI women. Apart from sex, the lesion level seems to affect fracture risk after SCI: patients with lumbar lesions have 2.4 times more fractures than those with cervical lesions. ${ }^{5}$

In the past, conservative fracture management measures such as pillow splints or bracing have been recommended. ${ }^{9-11}$ In this context, $87 \%$ of the extremity fractures documented in the Boston SCI cohort study have been treated conservatively. ${ }^{12}$ However more recently, operative fracture management has been advocated. ${ }^{2,13}$ Generally, the goal of fracture management is the restoration of the level of prefracture activity and functioning of the patient. ${ }^{2,4}$ As a consequence, an individual approach to fracture treatment has been recommended. ${ }^{11}$

Carbone et al. ${ }^{14}$ have observed post-fracture complications in $54 \%$ of SCI patients with lower-extremity fractures. Complications that commonly arise after a fracture are decubitus ulcers, venous thromboembolic events, mal-alignment, non-union or mal-union. ${ }^{14,15}$ To date, only few reports exist concerning the differences in fracture vulnerability between SCI subgroups. In addition, detailed information regarding fracture characteristics and the applied fracture treatment in daily clinical routine in a larger SCI cohort is scarce. It is unclear whether the extent of the neurological compromise affects fracture location, characteristics or recurrence of fractures. On the basis of this knowledge, individuals at risk of complex fractures could be identified and specific recommendations for the prevention of complex and recurrent long-bone fracture in persons with SCI could be established. Thus, the purpose of this study was to document long-bone fractures, fracture type according to the Müller AO classification, ${ }^{16}$ applied fracture management as well as fracture-related complications in individuals with a history of traumatic SCI. We also analysed whether the severity of long-bone fractures and the presence of

${ }^{1}$ Clinical Trial Unit, Swiss Paraplegic Centre, Nottwil, Switzerland; ${ }^{2}$ Department of Orthopaedics, Swiss Paraplegic Centre, Nottwil, Switzerland and ${ }^{3}$ Department of Osteoporosis, Inselspital, Berne University Hospital and University of Berne, Berne, Switzerland

Correspondence: Dr A Frotzler, Clinical Trial Unit, Swiss Paraplegic Centre, Guido A. Zäch-Strasse 1, 6207 Nottwil, Switzerland.

E-mail: angela.frotzler@paraplegie.ch

Received 22 December 2014; revised 1 April 2015; accepted 1 April 2015; published online 19 May 2015 
recurrent long-bone fracture events was affected by SCI-specific factors such as the American Spinal Injury Association Impairment Scale (AIS) ${ }^{17}$ and the lesion level of SCI.

\section{METHODS}

This retrospective study was conducted at a single centre specialized in the rehabilitation of SCI patients. Records of in- and out-patients between 2000 and 2011 were reviewed. Data of patients who met the following inclusion criteria were extracted: traumatic SCI and extremity fractures that occurred after the time point of SCI (corresponding to Class II or III according to McMaster and Stauffer ${ }^{11}$ ) and availability of at least baseline conventional radiographs after a long-bone fracture. The subsequent parameters were extracted from the patient records: patient characteristics (age, gender, AIS time since injury, lesion level (cervical, thoracic or lumbar SCI)), fractured bone, fracture localisation and severity according to the Müller AO classification of long-bone fractures, ${ }^{16}$ fracture management (operative versus conservative), implant type in case of operative treatment and type of fracture management in case of conservative treatment, fracture healing (complete versus incomplete) as well as occurrence of fracture-related complications. Fracture healing, evaluated on anteroposterior and lateral radiographs, was defined complete if the cortical bone was organised parallel to the axis of the bone, that is, if consolidation was achieved. Radiographs were routinely taken immediately after the fracture as well as 6 weeks, 3 months, 6 months and 1 year after the fracture, as clinically indicated.

This study has been approved by the Ethics Review Committee of the Canton Lucerne, Switzerland. All applicable institutional and governmental regulations concerning the ethical use of patient data were followed. Descriptive statistics were used to present data as frequencies with mean values and corresponding s.d. when normally distributed. In case of multiple long-bone fractures, the patients' characteristics at the time of the first-reported long-bone fracture were considered. The independent $t$-test was used to analyse differences between sex-specific patients' characteristics. The $\chi^{2}$-test (or exact Fisher's test where appropriate) was used to identify risk factors, that is, to test for significance SCI-specific factors, such as the AIS classification or SCI lesion level (dichotomised into paraplegia/tetraplegia) within the AO classification. ${ }^{16}$ The level of significance was set at $\alpha=0.05$. Statistical analyses were performed using the SPSS software (Version 18.0.3, IBM, Somers, NY, USA).

\section{RESULTS}

A total of 107 SCI patients (34 women and 73 men) fulfilled the inclusion criteria. Ninety-two patients $(86.0 \%)$ were classified as AIS A. The majority of the patients were paraplegics (77.6), and $72.0 \%$ of the paraplegic patients had an upper motor neuron lesion. Mean age at SCI was $30.5 \pm 13.8$ years and mean time since SCI at the time of the first-reported long-bone fracture was $20.1 \pm 12.2$ years. Women and men showed similar distributions of

Table 1 Patients' characteristics presented as frequencies or mean \pm s.d.

\begin{tabular}{|c|c|c|c|c|}
\hline Parameter & Overall & Women & Men & $\mathrm{P}$-value $\mathrm{a}^{\mathrm{a}}$ \\
\hline$n$ & 107 & 34 & 73 & \\
\hline AIS (A/B/C/D) & $92 / 9 / 4 / 2$ & $30 / 4 / 0 / 0$ & $62 / 5 / 4 / 2$ & 0.322 \\
\hline Paraplegia/tetraplegia & $83 / 24$ & $27 / 7$ & $56 / 17$ & 0.809 \\
\hline Age at $\mathrm{SCl}$ (years) & $30.5 \pm 13.8$ & $34.8 \pm 15.8$ & $28.5 \pm 12.3$ & $0.026^{b}$ \\
\hline $\begin{array}{l}\text { Age at long-bone fracture } \\
\text { (years) }\end{array}$ & $50.6 \pm 12.9$ & $53.4 \pm 14.3$ & $48.9 \pm 11.7$ & 0.056 \\
\hline Time since SCI (years) & $20.1 \pm 12.2$ & $18.7 \pm 10.9$ & $20.5 \pm 12.6$ & 0.623 \\
\hline
\end{tabular}

AIS classifications $(P=0.322)$, lesion levels (paraplegia/tetraplegia; $P=0.809)$ and cervical, thoracic or lumbar SCI $(P=0.684)$. Except for the age at SCI, men and women did not differ significantly with regard to age at first-reported long-bone fracture and time since injury (Table 1).

Altogether, 156 long-bone fractures were extracted from the medical records of the 107 included patients. A total of 70 patients $(65.4 \%)$ had one fracture, $28(26.1 \%)$ had two fractures, 7 patients $(6.5 \%)$ suffered from three fractures, 1 patient had four and 1 patient had five fractures. Concomitant fractures (that is, two fractures per event) occurred in 16 patients, all other multiple fractures were subsequent events. Only the lower extremities were affected, whereby the femur $(60.9 \%)$ was more frequently fractured than the lower leg $(39.1 \%)$. The distribution of the fracture localisation within a bone differed significantly between the upper and the lower leg $(P=0.035)$ : in the femur, most of the fractures $(44.7 \%)$ were distally located, followed by $29.8 \%$ proximal and $25.5 \%$ diaphyseal fractures (Table 2 and Figure 1). In the lower leg, fractures were distributed as follows: $41.9 \%$ proximal, $38.7 \%$ distal, $14.5 \%$ diaphyseal and $4.8 \%$ malleolar fractures (Table 2 and Figure 1). Overall, simple or extraarticular fractures (AO classification, A) were most frequent (75.0\%). Partial or complete articular fractures (AO classification, B) accounted for $17.3 \%$ of the fractures, and $7.7 \%$ were classified as complex or complete articular fractures (AO classification $=\mathrm{C}$; Table 2). The characterisation of fracture fragments differed significantly between the femur and the lower leg $(P=0.009)$.

Overall, $130(83.3 \%)$ fractures were treated operatively and 26 $(16.7 \%)$ conservatively. Femoral fractures $(n=83)$ were managed operatively significantly more often than lower leg $(n=47)$ fractures $(P=0.035)$. Approximately half of the femur fractures $(48.2 \%)$ were treated with locking compression plates. At the lower leg, fractures were mainly managed with external fixation (48.8\%; Figure 2). Conservative fracture management consisted of braces $(22$ cases) or a well-padded soft cast (four cases). The occurrence of fractureassociated complications did not differ significantly between operative and conservative fracture management $(P=0.940)$. Complications were present in 21 cases: $13.1 \%$ postoperative and $15.4 \%$ during conservative fracture management (Table 3 ). In one case, operative management of a wedge fracture at the distal lower leg was performed after conservative fracture treatment had failed. Fracture healing was complete in $64.7 \%$ of the cases within 1 year post fracture. In $9.6 \%$ (15 fractures), fracture healing was not complete and in $25.6 \%$ (40 fractures), fracture healing was not assessable due to either a too short radiographic follow-up period or the availability of baseline

Table 2 Distribution of fractures $(n=156)$ according to AO classification

\begin{tabular}{lccccccccccc}
\hline & & $A 1$ & $A 2$ & $A 3$ & $B 1$ & $B 2$ & $B 3$ & $C 1$ & $C 2$ & C3 & Total \\
\hline \multirow{2}{*}{ Femur } & 31 & 13 & 3 & 11 & & 1 & 1 & & 1 & & 30 \\
& 32 & 10 & 3 & 5 & 4 & 2 & & 1 & & & 25 \\
& 33 & 15 & 1 & 12 & 6 & 2 & & 3 & & & 39 \\
\multirow{5}{*}{ Lower leg } & 41 & 2 & 8 & 2 & 4 & 4 & & 3 & 2 & & 25 \\
& 42 & 5 & 3 & 1 & 1 & & & 1 & & 1 & 12 \\
& 43 & 14 & 3 & 5 & 1 & & & & & & 23 \\
& 44 & 1 & & & 1 & & & & & & 2 \\
& Total & 60 & 21 & 36 & 17 & 9 & 1 & 8 & 3 & 1 &
\end{tabular}

3, femur; 4, lower leg. 1, proximal; 2, diaphyseal; 3, distal; 4, malleolar. A, simple or extraarticular fracture; B, partial articular or wedge fracture; C, complete articular or complex fracture. 1,2 and 3 , severity of a fracture. The meaning of 1,2 and 3 differs for each bone and location. For convenience, the meaning of the numbers can be summarised as: 1 , simple; 2 , moderate; 3 , severe. 


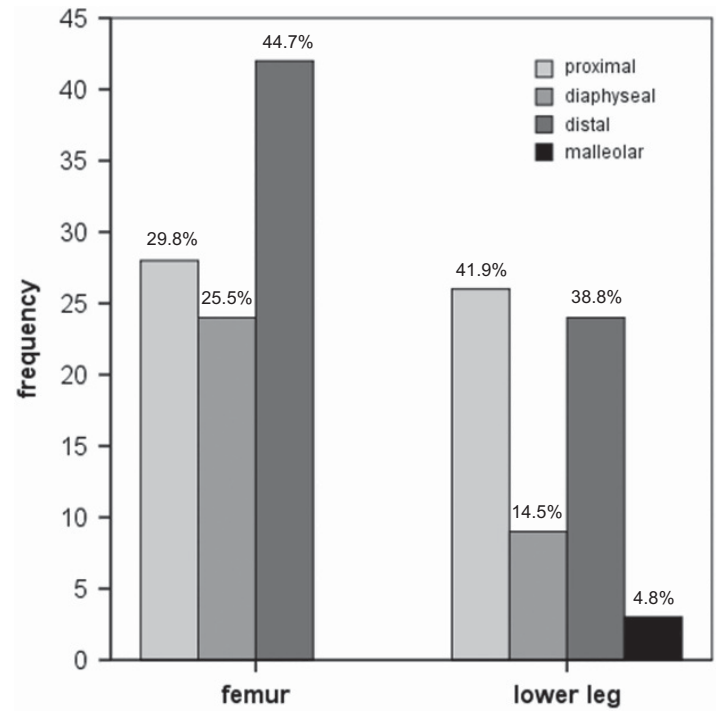

Figure 1 Distribution of fracture location within the bone, for the femur and the lower leg $(n=156)$. Percentages were calculated separately for the femur and the lower leg.

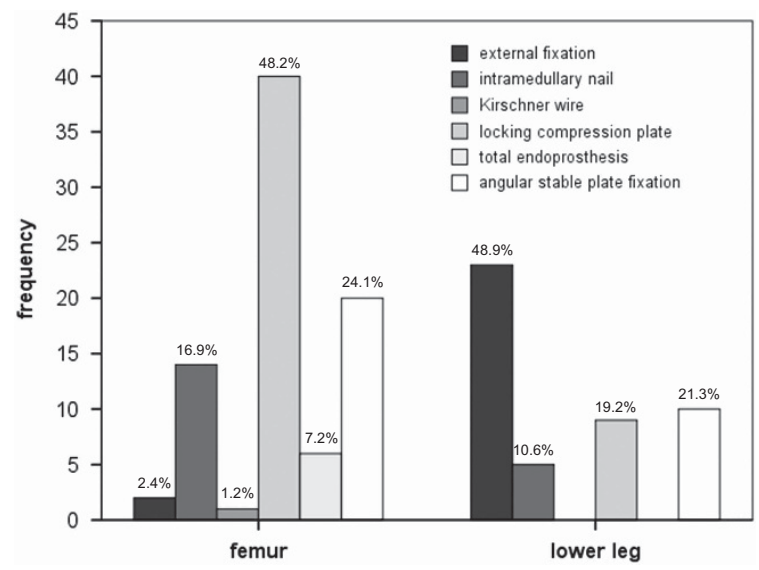

Figure 2 Number and type of operative fracture management $(n=130)$ for the femur and the lower leg. Percentages were calculated separately for the femur and the lower leg. Overall, surgical fracture management was applied in 83 femoral fractures and in 47 lower-leg fractures.

radiographs only. The rate of fracture healing did not differ significantly between operative and conservative fracture management $(P=0.120)$. Neither lesion level (cervical, thoracic or lumbar) nor AIS classification (A or B) nor sex had a significant effect on the characteristics of the long-bone fractures. Due to the small number of patients with an AIS C and D classification, statistical analyses were restricted to AIS A and B patients. However, there was a numerically higher proportion of femur fractures $(73.2 \%)$ vs lower-leg fractures $(26.8 \%)$ in patients with a lesion of the lower motor neuron compared with patients with an upper motor neuron lesion $(55.7 \%$ femur fractures vs $44.3 \%$ lower-leg fractures; $P=0.063$ ). Compared with patients with only one long-bone fracture event, patients with one or more subsequent fracture events had comparable distributions with regard to AIS classification $(P=0.493)$, sex $(P=0.192)$, lesion level (that is, paraplegia/tetraplegia; $P=0.334$ ) and upper or lower motor neuron lesion $(P=0.653)$.
Table 3 Type and count of complications after 156 long-bone fractures treated operatively and conservatively

\begin{tabular}{lcc}
\hline Complication & Operative $(\mathrm{n}=130)$ & Conservative $(\mathrm{n}=26)$ \\
\hline Non-union & 2 & 2 \\
Delayed union & 2 & 0 \\
Re-fracture & 6 & 0 \\
Infect & 4 & 0 \\
Mal-alignment & 0 & 1 \\
Treatment conversion & 1 & 1 \\
Re-osteosynthesis & 2 & 0 \\
Total & $17(13.1 \%)$ & $4(15.4 \%)$ \\
\hline
\end{tabular}

\section{DISCUSSION}

The characteristics of 156 long-bone fractures in 107 individuals with a history of traumatic SCI were studied retrospectively. The majority of the long-bone fractures were localised in the distal femur, followed by fractures of the proximal lower leg. No fractures of the upper limbs were recorded. Most of the fractures (75.0\%) were simple or extraarticular fractures. Overall, $83.3 \%$ of the fractures were treated operatively. Fracture-associated complications were present in $13.5 \%$ of the cases with similar complication rates after operative and conservative fracture management. With respect to fracture severity, no significant differences were found between patients with cervical, thoracic or lumbar SCI, between patients classified as AIS A or B and between men and women. Although AIS was not found to significantly influence fracture characteristics, it should be considered that $94 \%$ of the patients were classified AIS A or B.

The results of the present study confirm the findings of previous studies in SCI individuals, in which the majority of long-bone fractures was also observed in the lower extremities. ${ }^{3,18}$ Several authors suggested that the preferential localisation in the lower extremities was a consequence of the dramatic sublesional decrease of bone mineral density in the lower leg following SCI. ${ }^{1,5,6,19,20}$ However, it is noteworthy that we observed more femoral than lower-leg fractures, although in individuals with motor complete SCI the loss of bone mineral density is known to be more pronounced in the latter. ${ }^{8,21}$ The fracture event itself and the resulting bending, torsion and compression forces are also important aspects that may account for the higher number of long-bone fractures in the femur than in the lower leg. Information concerning the magnitude of bone loss is missing in the present study because osteodensitometry was not routinely performed in these patients in our institution. However, as $86.0 \%$ of the investigated patients suffered from chronic motor complete SCI, with a mean time of $\sim 20$ years from injury to the first-reported fracture, it may be assumed that most of our patients suffered from severe regional osteoporosis at the time of long-bone fracture, consistent with earlier observations reporting that bone mineral density in the lower extremities of individuals with chronic SCI was reduced by $50-70 \% 0^{7,8}$ with a median femoral neck $T$-score of -3.9 corresponding to severe osteoporosis. $^{18}$

Approximately 35\% of the investigated patients had experienced two or more subsequent long-bone fracture events. This proportion is slightly higher than the $28 \%$ reported by Fatal et al. ${ }^{18}$ In the SCI population, and in contrast to the general population, fracture risk and the number of subsequent long-bone fractures appear to be more related to time since SCI than age. ${ }^{1,3,10}$ So far, several pharmacologic and non-pharmacologic interventions aimed at reducing bone loss and/or at increasing bone strength after SCI have been investigated. ${ }^{22,23}$ However, the effect of these interventions with 
regard to the reduction of long-bone fracture risk remains unclear. Thus, preventative measures should be taken and the awareness of the increased risk of long-bone fractures should be raised among patients early after SCI and among medical staff.

Consistent with other reports, ${ }^{1,13}$ fracture localisation within the bone differed significantly between the upper and the lower leg. Approximately $44 \%$ of the femur fractures were located distally in comparison to the lower leg, where fractures occurred both proximally and distally. With respect to fracture severity, our findings parallel the results of Bärlehner et al. ${ }^{2}$ who classified 41 out of 55 (75\%) long-bone fractures in patients with chronic SCI as 'simple' or 'extraarticular' according to the AO classification. This fracture distribution may reflect the common mechanism of fracture events in individuals with SCI, which are often the result of falls out of the wheelchair during transfers or propelling. ${ }^{24}$

In contrast to previous recommendations for a conservative treatment of long-bone fracture in SCI individuals, ${ }^{3,9-11}$ most of the long-bone fractures were treated operatively in the present cohort. Femur fractures were mainly managed with locking compression plates, whereas lower-leg fractures were mainly treated with external fixation. The rationale for the operative management of long bones in paralysed limbs is based on the early return to previous activities and high patient satisfaction. ${ }^{2,13}$ However, strong recommendations concerning operative or conservative treatment of long-bone fractures in SCI individuals cannot be drawn from our data, due to the retrospective study design. For this purpose, prospective controlled interventional studies would be required.

In contrast to previous studies with a reported complication rate after long-bone fractures of between 19 and 54\%, 4,12,14 our work showed a lower complication rate of $\sim 14 \%$, with a similar complication rate for operative and conservative fracture management. An explanation to this finding might be that our institution is specialised in the rehabilitation of patients with SCI and thus, the medical staff is very experienced with the management and care of long-bone fractures. Delayed fracture healing was observed in $\sim 10 \%$ of the cases. According to our experience, delayed fracture healing can result in prolonged immobilisation with an increased risk of pressure sores, general infections and prolonged hospitalisation. In addition, a delayed union may give rise to surgical revisions or interventions and thus increased costs.

In addition to its retrospective nature, our study has some limitations. Investigated fractures were not necessarily the first fractures after traumatic SCI and thus, the reported time to long-bone fracture after SCI may have been overestimated. On the other hand, the number of reported fractures within the observation period may have been underestimated, as all included fractures were radiographically documented. The collection of fracture-related complications was limited to dichotomised data (yes or no). Furthermore, clinically relevant data such as range of motion, patient's satisfaction, duration of hospital stay and use of bone-active drugs or calcium and vitamin D are missing. Finally, the detailed indications for operative or conservative fracture management were not available from the patients' records. However, this study presents an overview of the pattern of long-bone fractures and their management in individuals with traumatic SCI in daily clinical routine in a specialized centre for paraplegics.

\section{CONCLUSION}

SCI is associated with simple or extraarticular long-bone fractures of the lower extremities, that is, distal femur and proximal or distal lower leg. The extent of the neurological compromise does not seem to affect the pattern of long-bone fractures. Multiple subsequent fracture events seem to be common. In our daily clinical routine, fractures were mainly treated operatively. The complication rate was comparably low both after operative and conservative fracture management.

\section{DATA ARCHIVING}

There were no data to deposit.

\section{CONFLICT OF INTEREST}

The authors declare no conflict of interest.

\section{ACKNOWLEDGEMENTS}

We would like to gratefully acknowledge the assistance of Kathrin Hartmann and Stefanie Tesini, from the Swiss Paraplegic Centre, with the data management.

1 Zehnder $\mathrm{Y}$, Luthi M, Michel D, Knecht $\mathrm{H}$, Perrelet R, Neto I et al.. Long-term changes in bone metabolism, bone mineral density, quantitative ultrasound parameters, and fracture incidence after spinal cord injury: a cross-sectional observational study in 100 paraplegic men. Osteoporos Int 2004; 15: 180-189.

2 Bärlehner C, Bohm V, Flieger R, Meiners T. [Surgery for fractures of the lowe extremities in cases of chronic spinal cord injury]. Orthopade 2005; 34:, 140-133.

3 Comarr $\mathrm{E}$, Hutchinson $\mathrm{RH}$, Bors $\mathrm{E}$. Extremity fractures of patients with spinal cord injuries. Top Spinal Cord Inj Rehabil 2005; 11: 1-10.

4 Cochran TP, Bayley JC, Smith M. Lower extremity fractures in paraplegics: pattern, treatment, and functional results. J Spinal Disord 1988; 1: 219-223.

5 Vestergaard P, Krogh K, Rejnmark L, Mosekilde L. Fracture rates and risk factors for fractures in patients with spinal cord injury. Spinal Cord 1998; 36: 790-796.

6 Eser P, Frotzler A, Zehnder Y, Denoth J. Fracture threshold in the femur and tibia of people with spinal cord injury as determined by peripheral quantitative computed tomography. Arch Phys Med Rehabil 2005; 86: 498-504.

7 Biering-Sorensen F, Bohr HH, Schaadt OP. Longitudinal study of bone mineral content in the lumbar spine, the forearm and the lower extremities after spinal cord injury. Eur J Clin Invest 1990; 20: 330-335.

8 Eser $\mathrm{P}$, Frotzler A, Zehnder Y, Wick L, Knecht H, Denoth J et al. Relationship between the duration of paralysis and bone structure: a $\mathrm{pQCT}$ study of spinal cord injured individuals. Bone 2004; 34: 869-880.

9 Freehafer AA. Limb fractures in patients with spinal cord injury. Arch Phys Med Rehabil 1995; 76: 823-827

10 Ingram RR, Suman RK, Freeman PA. Lower limb fractures in the chronic spinal cord injured patient. Paraplegia 1989; 27: 133-139.

11 McMaster WC, Stauffer ES. The management of long bone fracture in the spinal cord injured patient. Clin Orthop Relat Res 1975, 44-52.

12 Morse LR, Battaglino RA, Stolzmann KL, Hallett LD, Waddimba A, Gagnon D et al. Osteoporotic fractures and hospitalization risk in chronic spinal cord injury. Osteoporos Int 2009; 20: 385-392.

13 Sugi MT, Davidovitch R, Montero N, Nobel T, Egol KA. Treatment of lower-extremity long-bone fractures in active, nonambulatory, wheelchair-bound patients. Orthopedics 2012; 35: e1376-e1382.

14 Carbone LD, Chin AS, Burns SP, Svircev JN, Hoenig H, Heggeness M et al.. Morbidity following lower extremity fractures in men with spinal cord injury. Osteoporos Int 2013; 24: 2261-2267.

15 Nottage WM. A review of long-bone fractures in patients with spinal cord injuries. Clin Orthop Relat Res 1981; 155: 65-70.

16 Müller AO. Classification of fractures-long bones. AOTrauma 2010.

17 Kirshblum SC, Waring W, Biering-Sorensen F, Burns SP, Johansen M, Schmidt-Read M et al. Reference for the 2011 revision of the International Standards for Neurological Classification of Spinal Cord Injury. J Spinal Cord Med 2011; 34: 547-554.

18 Fattal C, Mariano-Goulart D, Thomas E, Rouays-Mabit H, Verollet C, Maimoun L et al. Osteoporosis in persons with spinal cord injury: the need for a targeted therapeutic education. Arch Phys Med Rehabil 011; 92: 59-67.

19 Biering-Sorensen $\mathrm{F}$, Bohr H, Schaadt O. Bone mineral content of the lumbar spine and lower extremities years after spinal cord lesion. Paraplegia 1988; 26: 293-301.

20 Garland DE. Fracture threshold and risk for osteoporosis and pathologic fractures in individuals with spinal cord injury. Top Spinal Cord Inj Rehabil 2005; 11: 61-69.

21 Coupaud S, McLean AN, Allan DB. Role of peripheral quantitative computed tomography in identifying disuse osteoporosis in paraplegia. Skeletal Radiol 2009; 38 989-995.

22 Biering-Sorensen F, Hansen B, Lee BS. Non-pharmacological treatment and prevention of bone loss after spinal cord injury: a systematic review. Spinal Cord 2009; 47 508-518.

23 Zehnder $\mathrm{Y}$, Risi S, Michel D, Knecht $\mathrm{H}$, Perrelet $\mathrm{R}$, Kraenzlin $\mathrm{M}$ et al. Prevention of bone loss in paraplegics over 2 years with alendronate. J Bone Miner Res 2004; 19: 1067-1074.

24 Nelson A, Ahmed S, Harrow J, Fitzgerald S, Sanchez-Anguiano A, Gavin-Dreschnack D et al. Fall-related fractures in persons with spinal cord impairment: a descriptive analysis. SCI Nurs Spring 2003; 20: 30-37. 